\title{
Method for Estimating the Dielectric Constant of Natural Gas Mixtures ${ }^{1}$
}

\author{
A. H. Harvey ${ }^{2,3}$ and E. W. Lemmon ${ }^{2}$
}

\begin{abstract}
A method has been developed for calculating the static dielectric constant (relative permittivity) of fluid mixtures, with an emphasis on natural gas. The dielectric constant is calculated as a function of temperature, density, and composition; the density is calculated with a fundamental mixture equation of state. Theory-based correlations were developed for the dielectric constant of all significant components of natural gas, including not only light hydrocarbons but also gases such as nitrogen and carbon dioxide. In many cases, these correlations took advantage of new, highly accurate data measured in cross capacitors. For mixtures, the pure-component values are combined as proposed by Harvey and Prausnitz; this produces better results than the traditional mixing rule.
\end{abstract}

KEY WORDS: dielectric constant; energy; mixtures; natural gas; polarization; relative permittivity.

\section{INTRODUCTION}

Natural gas is an increasingly important energy source. There is interest in methods, based on relatively simple measurements, for estimating important properties such as the heating value. It has been proposed $[1,2]$ that measurements of the static dielectric constant could be a part of such a method.

In order to evaluate and optimize such methods, a procedure is needed for accurate calculations of the dielectric constant of natural gas as

\footnotetext{
${ }^{1}$ Paper presented at the Fifteenth Symposium on Thermophysical Properties, June 22-27, 2003, Boulder, Colorado, U.S.A.

${ }^{2}$ Physical and Chemical Properties Division, National Institute of Standards and Technology, 325 Broadway, Boulder, Colorado 80305-3328, U.S.A.

${ }^{3}$ To whom correspondence should be addressed. E-mail: aharvey@boulder.nist.gov
} 
a function of temperature, pressure, and composition. While correlations exist [3] for the dielectric constant of the main components of natural gas, the availability of new experimental data of high quality [4,5] (and the development of better equations of state for these components) affords the opportunity for significant improvement. In addition, the pure-component values must be combined in a fundamentally sound manner to obtain accurate dielectric constants of mixtures.

In this work, we first produce correlations for the static dielectric constant of various pure components; these correlations are based on theoretical considerations to the extent possible. We then apply a previously proposed mixing rule [6], and show that it produces good results for mixtures.

\section{PURE-COMPONENT CORRELATIONS}

\subsection{Theoretical Background}

Theory and modeling for the static dielectric constant $\varepsilon$ typically do not use $\varepsilon$ itself, but rather the electric polarization $P$. While there is no universally valid relationship between $P$ and $\varepsilon$, a good approximation for nonpolar fluids is the Clausius-Mosotti expression,

$$
P_{\mathrm{CM}}=\frac{\varepsilon-1}{\varepsilon+2} .
$$

For polar fluids, an expression due to Kirkwood is often used;

$$
P_{\mathrm{K}}=\frac{(\varepsilon-1)(2 \varepsilon+1)}{9 \varepsilon} .
$$

Equations (1) and (2) have the same limiting behavior for small values of $\varepsilon-1$, but differ increasingly for larger $\varepsilon$. More complicated expressions for polar fluids have been proposed, usually involving a separate "infinitefrequency" dielectric constant; the book by Böttcher [7] provides details.

For nonpolar fluids, the polarization divided by the molar density $\rho$ is constant to a first approximation, and it is common to expand $P / \rho$ in a power series known as the dielectric virial expansion:

$$
P / \rho=A_{\varepsilon}+B_{\varepsilon} \rho+C_{\varepsilon} \rho^{2}+\cdots .
$$

$A_{\varepsilon}$, the first dielectric virial coefficient, is directly proportional to the molecular static polarizability $\alpha$. For molecules with internal structure, $\alpha$ (and therefore $A_{\varepsilon}$ ) has a slight temperature dependence. This is because higher temperature causes some molecules to be in higher rotational states (causing centrifugal stretching) and/or higher vibrational states (altering 


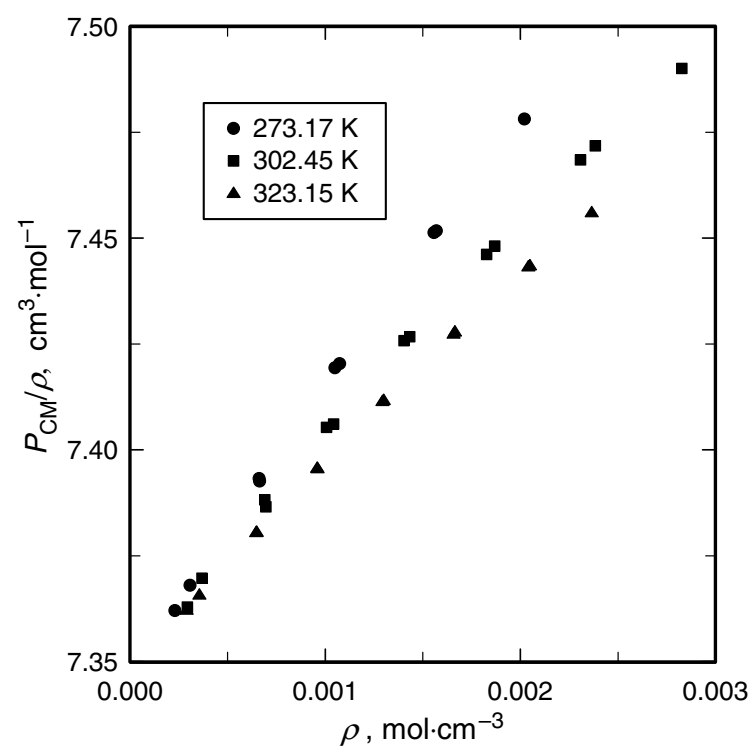

Fig. 1. Illustration of temperature dependence of the slope $\left(B_{\varepsilon}\right)$ of the molar polarization of $\mathrm{CO}_{2}$. Data from Moldover and Buckley [4] (323.15 K) and Schmidt and Moldover [5] (273.17 and $302.45 \mathrm{~K})$.

average bond lengths due to anharmonicity). For the molecules and temperatures of interest here, the rotational effect is most important; this produces an approximately linear increase of $A_{\varepsilon}$ with temperature [8].

$B_{\varepsilon}$, the second dielectric virial coefficient, is due to interactions between pairs of molecules. For molecules without permanent dipoles, the dominant influence on $B_{\varepsilon}$ is usually the higher multipoles (quadrupole, octupole, etc.). These result in values of $B_{\varepsilon}$ that are approximately linear in $1 / T$, where $T$ is the absolute temperature [9]. Figure 1 illustrates this inverse temperature dependence in $B_{\varepsilon}$ for recent precise low-density data $[4,5]$ for $\mathrm{CO}_{2}$, whose large quadrupole moment leads to a relatively large effect.

For molecules with a permanent dipole moment $\mu$, an additional term contributes to the low-density expansion of the molar polarization:

$$
P / \rho=A_{\varepsilon}+\frac{N_{\mathrm{A}} \mu^{2}}{9 \varepsilon_{0} k_{\mathrm{B}} T}+B_{\varepsilon} \rho+C_{\varepsilon} \rho^{2}+\cdots,
$$

where $N_{\mathrm{A}}$ is Avogadro's constant, $\varepsilon_{0}$ is the electric constant (permittivity of free space), and $k_{\mathrm{B}}$ is Boltzmann's constant. 
Table I. Literature Sources for Pure-Component DielectricConstant Data and Equations of State (EOS)

\begin{tabular}{lll}
\hline Fluid & \multicolumn{1}{c}{ Dielectric data } & EOS \\
\hline $\mathrm{He}$ & {$[4,5,10]$} & {$[11]$} \\
$\mathrm{Ne}$ & {$[10,12,13]$} & {$[14]$} \\
$\mathrm{Ar}$ & {$[4,5,10,15,16]$} & {$[17]$} \\
$\mathrm{Kr}$ & {$[12,18,19]$} & {$[20]$} \\
$\mathrm{Xe}$ & {$[12,18,21]$} & {$[20]$} \\
$\mathrm{H}_{2}$ & {$[22]$} & {$[23]$} \\
$p-\mathrm{H}_{2}$ & {$[22]$} & {$[23]$} \\
$\mathrm{N}_{2}$ & {$[4,5,15,16,24]$} & {$[25]$} \\
$\mathrm{O}_{2}$ & {$[5,26]$} & {$[27]$} \\
$\mathrm{CO}_{2}$ & {$[4,5,28-30]$} & {$[31]$} \\
$\mathrm{CH}_{4}$ & {$[4,5,15,16,29,32-35]$} & {$[36]$} \\
$\mathrm{C}_{2} \mathrm{H}_{4}$ & {$[37,38]$} & {$[39]$} \\
$\mathrm{C}_{2} \mathrm{H}_{6}$ & {$[5,40]$} & {$[41]$} \\
$\mathrm{C}_{3} \mathrm{H}_{8}$ & {$[5,30,42-45]$} & {$[46]$} \\
$n-\mathrm{C}_{4} \mathrm{H}_{10}$ & {$[44,47,48]$} & {$[49]$} \\
$i-\mathrm{C}_{4} \mathrm{H}_{10}$ & {$[44,47,50]$} & {$[49]$} \\
$n-\mathrm{C}_{5} \mathrm{H}_{12}$ & {$[51]$} & {$[52]$} \\
$i-\mathrm{C}_{5} \mathrm{H}_{12}$ & {$[43,53]$} & {$[20]$} \\
$n-\mathrm{C}_{6} \mathrm{H}_{14}$ & {$[51]$} & {$[52]$} \\
$n-\mathrm{C}_{7} \mathrm{H}_{16}$ & {$[51]$} & {$[52]$} \\
$n-\mathrm{C}_{8} \mathrm{H}_{18}$ & {$[51]$} & {$[52]$} \\
$n-\mathrm{C}_{9} \mathrm{H}_{20}$ & {$[51]$} & {$[20]$} \\
$n-\mathrm{C}_{10} \mathrm{H}_{22}$ & {$[51]$} & {$[20]$} \\
\hline
\end{tabular}

\subsection{Data Sources and Treatment}

In Table I, we list the sources of pure-component dielectric-constant data used in fitting our correlations. In some cases, additional sources were examined but were rejected because their data were outliers or because data of higher quality were available covering the same range of conditions.

For nonpolar substances, the dielectric data were converted to $P_{\mathrm{CM}}$ with Eq. (1). For those with a nonzero dipole moment, both $P_{\mathrm{CM}}$ and $P_{\mathrm{K}}$ (Eq. (2)) were derived, as the final correlations (see below) sometimes used one and sometimes the other.

Because the quantity ultimately correlated was $P / \rho$, while the dielectric data were reported as a function of temperature $T$ and pressure $p$, equations of state were required to calculate $\rho(p, T)$ for each point. These calculations were performed with the NIST REFPROP database [54], which uses the best available pure-component equations of state. Table I lists the references for the equations of state used. 


\subsection{Dipole Moments}

Dipole moments were taken from the compilation of Nelson et al. [55] for propane $\left(0.084 \mathrm{D} ; 1 \mathrm{D} \approx 3.335641 \times 10^{-30} \mathrm{C} \cdot \mathrm{m}\right)$ and isobutane $(0.132 \mathrm{D})$. A complication arises for molecules that exist in multiple conformations, each having a different dipole moment. For example, $n$-butane can exist in a trans form (with zero dipole moment, due to symmetry) or a gauche form (whose dipole moment has been measured [56] as 0.09 D). The resulting temperature-dependent expectation value of $\mu$ can be determined by a Boltzmann-weighted average over the conformations. Rigorously, such a calculation should take into account not only the energy difference between the conformers, but also differences in the partition functions of the isolated molecules. Because the latter entropic effect is relatively small, and because the quality of our correlations is insensitive to small changes in the dipole moment, we used only the energy difference [57] in our calculations.

While one could, in principle, include the entire temperature-dependent calculation of the dipole moment in the correlation, the changes with temperature are not large and the required accuracy for these minor components does not justify the extra complication. Therefore, we use the value at a single temperature, which we choose as the normal boiling point to be consistent with the way we tabulate dipole moments elsewhere [54]. Because of the approximations in our procedure, we round the value to the nearest $0.01 \mathrm{D}$. For $n$-butane, the resulting value is $0.05 \mathrm{D}$. For $n$-pentane, which has four stable conformers, we use energy differences [58] and dipole moments [59] from ab initio calculations, resulting in an averaged dipole moment of $0.07 \mathrm{D}$ at the normal boiling point. Similar calculations [60] for isopentane (methyl butane) yield an averaged value of $0.11 \mathrm{D}$.

In principle, the same procedure could be used for longer alkanes, but the number of conformations to consider rapidly becomes unwieldy. Since longer alkanes are only trace components in natural gas, since the available data are limited, and since small differences in $\mu$ can be absorbed by other parameters in the correlation, we approximate the dipole moment for $n$-hexane and all higher $n$-alkanes by the $n$-pentane value of $0.07 \mathrm{D}$.

\subsection{Data Correlation}

The form of the correlation was based on the dielectric virial expansion (Eq. (4)), with temperature dependences of $A_{\varepsilon}$ and $B_{\varepsilon}$ as discussed in Section 2.1. $A_{\varepsilon}$ and to a lesser extent $B_{\varepsilon}$ are the most important terms for describing gas-phase data, which are of primary interest in this work. Because higher-order terms are difficult to extract from data, an empirical 
form was chosen to extend the correlation to high densities. The final form of the correlation is

$$
P / \rho=A_{\varepsilon}+A_{\mu} / T+B_{\varepsilon} \rho+C \rho^{D},
$$

where $A_{\mu}=\frac{N_{\mathrm{A}} \mu^{2}}{9 \varepsilon_{0} k_{\mathrm{B}}}$ is a compact way of writing factors in the dipolar term of Eq. (4) and is not an adjustable parameter. The virial coefficients $A_{\varepsilon}$ and $B_{\varepsilon}$ and the parameter $C$ are made temperature-dependent as follows:

$$
\begin{aligned}
& A_{\varepsilon}=a_{0}+a_{1}\left(\frac{T}{T_{0}}-1\right) \\
& B_{\varepsilon}=b_{0}+b_{1}\left(\frac{T_{0}}{T}-1\right) \\
& C=c_{0}+c_{1}\left(\frac{T_{0}}{T}-1\right) .
\end{aligned}
$$

The temperature dependence of $A_{\varepsilon}$ and $B_{\varepsilon}$ follow the theoretical results discussed in Section 2.1, while that of parameter $C$ is empirical. The exponent $D$ is temperature-independent. $T_{0}$ is an arbitrary reference temperature; following previous work [5], we choose $T_{0}=273.16 \mathrm{~K}$.

In fitting Eq. (5), large weight was given to recent high-quality gasphase data $[4,5]$ where they were available. Emphasis was placed on accuracy at gas-like densities; liquid-phase data were included in the fits but were not always fitted within their experimental uncertainties. In most cases, the gas-phase data were examined in isolation to determine $A_{\varepsilon}\left(a_{0}\right.$ and $a_{1}$ ); these were then held constant while the remaining parameters were fitted to the full data set.

For the left-hand side of Eq. (5), the Clausius-Mosotti form of $P / \rho$ (Eq. (1)) was used for nonpolar components. Some components of natural gas (such as propane) have a small dipole moment. For those components, both the Kirkwood (Eq. (2)) and Clausius-Mosotti forms were tried, and the one that gave the best overall fit was used. In Table II, we report the correlation parameters; for all fluids but propane the fits used the Clausius-Mosotti form. The use of Eq. (5) with these parameters requires densities $\rho$ to be in $\mathrm{mol} \cdot \mathrm{cm}^{-3}$.

Because Eq. (5) is a function of density, the fitted parameters are linked to the equation of state for the pure fluid. For some fluids (He and $\mathrm{H}_{2}$, for example), the uncertainty in the EOS is substantial, and if a better EOS is developed, it would be advisable to repeat the data reduction and fitting process. For other fluids where the uncertainties in the EOS 
Table II. Parameters for Correlation of Molar Polarization with Eq. (5). (Units: $a_{0}$ and $a_{1}$ in $\mathrm{cm}^{3} \cdot \mathrm{mol}^{-1} ; A_{\mu}$ in $\mathrm{cm}^{3} \cdot \mathrm{mol}^{-1} \cdot \mathrm{K} ; b_{0}$ and $b_{1}$ in $\mathrm{cm}^{6} \cdot \mathrm{mol}^{-2} ; c_{0}$ and $c_{1}$ in $\left.\mathrm{cm}^{3(D+1)} \cdot \mathrm{mol}^{-(D+1)}\right)$

\begin{tabular}{|c|c|c|c|c|c|c|c|c|}
\hline Fluid & $a_{0}$ & $a_{1}$ & $A_{\mu}$ & $b_{0}$ & $b_{1}$ & $c_{0}$ & $c_{1}$ & $D$ \\
\hline $\mathrm{He}$ & 0.517254 & 0 & 0 & -0.203 & 0.039 & 7.47 & 0 & 2 \\
\hline $\mathrm{Ne}$ & 0.9969 & 0 & 0 & -0.109 & 0.0708 & -2.88 & -1.0 & 2 \\
\hline $\mathrm{Ar}$ & 4.1414 & 0 & 0 & 1.597 & 0.262 & -117.9 & 0 & 2.1 \\
\hline $\mathrm{Kr}$ & 6.273 & 0 & 0 & 6.485 & 13.48 & -82.51 & -170.4 & 1.7 \\
\hline $\mathrm{Xe}$ & 10.122 & 0 & 0 & 31.97 & 46.97 & -948.4 & 0 & 1.7 \\
\hline $\mathrm{H}_{2}$ & 2.0306 & 0.0056 & 0 & 0.181 & 0.021 & -7.4 & 0 & 2 \\
\hline$p-\mathrm{H}_{2}$ & 2.0297 & 0.0069 & 0 & 0.181 & 0.021 & -7.4 & 0 & 2 \\
\hline $\mathrm{N}_{2}$ & 4.3872 & 0.00226 & 0 & 2.206 & 1.135 & -169.0 & -35.83 & 2.1 \\
\hline $\mathrm{O}_{2}$ & 3.9578 & 0.0065 & 0 & 0.575 & 1.028 & -8.96 & -5.15 & 1.5 \\
\hline $\mathrm{CO}_{2}$ & 7.3455 & 0.00335 & 0 & 83.93 & 145.1 & -578.8 & -1012 & 1.55 \\
\hline $\mathrm{CH}_{4}$ & 6.5443 & 0.0133 & 0 & 8.4578 & 3.7196 & -352.97 & -100.65 & 2 \\
\hline $\mathrm{C}_{2} \mathrm{H}_{4}$ & 10.725 & 0 & 0 & 55.19 & 49.5 & -2045 & -1154 & 1.9 \\
\hline $\mathrm{C}_{2} \mathrm{H}_{6}$ & 11.1552 & 0.0112 & 0 & 36.759 & 23.639 & -808.03 & -378.84 & 1.75 \\
\hline $\mathrm{C}_{3} \mathrm{H}_{8}^{a}$ & 15.850 & 0.036 & 42.97 & 172.75 & 505.67 & -388.21 & -2078.8 & 1.35 \\
\hline$n-\mathrm{C}_{4} \mathrm{H}_{10}$ & 20.611 & 0.020 & 15.23 & 66.64 & 24.44 & -7461.2 & -1983.6 & 2 \\
\hline$i-\mathrm{C}_{4} \mathrm{H}_{10}$ & 20.534 & 0.020 & 106.1 & 126.25 & 52.91 & -7501.4 & -2672.9 & 1.9 \\
\hline$n-\mathrm{C}_{5} \mathrm{H}_{12}$ & 25.39 & 0.025 & 29.84 & 78.39 & 54.15 & -12480 & -4800.0 & 2 \\
\hline$i-\mathrm{C}_{5} \mathrm{H}_{12}$ & 25.31 & 0.025 & 73.69 & 108.9 & 63.68 & -15447 & -5449.3 & 2 \\
\hline$n-\mathrm{C}_{6} \mathrm{H}_{14}$ & 30.18 & 0.030 & 29.84 & 222.31 & 232.62 & -36872 & -25733 & 2 \\
\hline$n-\mathrm{C}_{7} \mathrm{H}_{16}$ & 34.96 & 0.035 & 29.84 & 162.24 & 308.90 & -37446 & -39684 & 2 \\
\hline$n-\mathrm{C}_{8} \mathrm{H}_{18}$ & 39.74 & 0.040 & 29.84 & 348.01 & 494.18 & -76838 & -65772 & 2 \\
\hline$n-\mathrm{C}_{9} \mathrm{H}_{20}$ & 44.53 & 0.045 & 29.84 & 286.27 & 529.31 & -83471 & -90493 & 2 \\
\hline$n-\mathrm{C}_{10} \mathrm{H}_{22}$ & 49.32 & 0.050 & 29.84 & 220.15 & -316.3 & -88358 & 53511 & 2 \\
\hline
\end{tabular}

${ }^{a}$ Equation (5) fit to $P_{\mathrm{K}} / \rho$ for $\mathrm{C}_{3} \mathrm{H}_{8}$; all others fit to $P_{\mathrm{CM}} / \rho$.

are already quite small, the error introduced by using the parameters in Table II with a different EOS of similar or better quality would be less significant.

\subsection{Discussion of Individual Systems}

Because of their scientific interest, the noble gases were included in this work, even though only $\mathrm{He}$ is significant in natural gas. Because the noble gases lack internal structure, $A_{\varepsilon}$ is independent of temperature. For $\mathrm{He}, A_{\varepsilon}$ is known with high accuracy from theory [61], so that value was used in preference to experimental results. While there are low-density data $[4,5,12]$ (to determine $A_{\varepsilon}$ and $B_{\varepsilon}$ ) for the noble gases, our highdensity results are based on limited data and should be viewed with less confidence. 


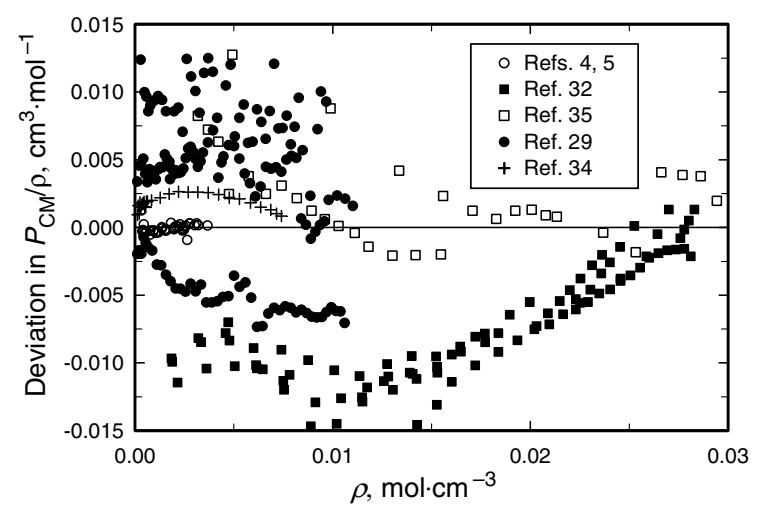

Fig. 2. Deviations of experimental data from Eq. (5) fit for methane.

For $\mathrm{H}_{2}$, data at low densities are lacking, and $A_{\varepsilon}$ was found from the ab initio polarizabilities of Rychlewski [62] for various rotational quantum states, where the populations of the states were calculated as a function of temperature from the partition function and known rotational constants. Because of the scarcity of data for $\mathrm{H}_{2}$, the remainder of the parameters in Eq. (5) were developed based on the data of Stewart [22] for parahydrogen. While $p-\mathrm{H}_{2}$ is not present in natural gas, we include a correlation for it here to replace a previous correlation [23]; the values for $A_{\varepsilon}$ once again were determined from ab initio polarizabilities [62].

For $\mathrm{N}_{2}, \mathrm{O}_{2}, \mathrm{CO}_{2}, \mathrm{CH}_{4}$, and $\mathrm{C}_{2} \mathrm{H}_{6}$, there are excellent low-density data $[4,5]$ that allow precise determination of $A_{\varepsilon}$, including its small temperature dependence. These fluids also have substantial data at higher densities, and reference-quality equations of state for determining $\rho(p, T)$, so they provide the most complete tests for our correlation. The low-density data for $P / \rho$ are reproduced to within $0.01 \%$ (comparable to their scatter) for $\mathrm{O}_{2}$ and $\mathrm{CH}_{4}$ and within $0.02 \%$ for $\mathrm{N}_{2}, \mathrm{CO}_{2}$, and $\mathrm{C}_{2} \mathrm{H}_{6}$. At liquid-like densities, the deviations are generally within $0.2 \%$; this is somewhat more than the scatter in the data and one can sometimes see small systematic trends. For $\mathrm{N}_{2}$, there are recent low-density data [63,64] for which $P / \rho$ is systematically high by about $0.1 \%$ compared to the low-density data on which we relied [4,5]; we have no explanation for this disagreement. For $\mathrm{CO}_{2}$, the saturated-liquid data of Haynes [65] were not used; May et al. [30] have recently made a strong case that the Haynes data for $\mathrm{CO}_{2}$ have a systematic error of about $0.5 \%$.

Because of the importance of methane in natural gas, we show in Fig. 2 the differences between experimental and calculated values of $P / \rho$ 
for $\mathrm{CH}_{4} . P / \rho$ is near $6.5 \mathrm{~cm}^{3} \cdot \mathrm{mol}^{-1}$, so the maximum deviations in Fig. 2 correspond to about $0.2 \%$ on a relative basis. For clarity, we omit some additional liquid-phase data $[15,16,33]$ (these are generally fitted to within $0.005 \mathrm{~cm}^{3} \cdot \mathrm{mol}^{-1}$ in $\left.P / \rho\right)$ and a few high-pressure points [35] at densities above $0.03 \mathrm{~mol} \cdot \mathrm{cm}^{-3}$ (these are fitted to within $0.003 \mathrm{~cm}^{3} \cdot \mathrm{mol}^{-1}$ ). It should be noted that temperature is an additional variable; for simplicity we plot all temperatures on the same graph. Figure 2 illustrates the differences in low-density data among various sources; we chose to use the recent data taken in cross capacitors $[4,5]$ because of their consistency and precision and because this apparatus produced data for He that agree with the value of $A_{\varepsilon}$ dictated by theory. At higher densities, there is a mild disagreement between the data of Malbrunot et al. [35] (which are only at $298 \mathrm{~K}$ ) and those of Straty and Goodwin [32] (which range from 91 to $300 \mathrm{~K}$ ). It is likely that the liquid data of Straty and Goodwin could be fit better at the expense of introducing more complicated temperature dependence, but, since cryogenic liquids are not our main concern, we chose not to add such complexity.

For $\mathrm{C}_{2} \mathrm{H}_{4}, A_{\varepsilon}$ and $B_{\varepsilon}$ were based entirely on the results of Bose and Cole [37], and the $C$ term was adjusted to fit the available liquid-phase data [38]. The liquid data were fitted to within $0.05 \%$, but that probably overestimates the reliability of the correlation because the data consist of only eight saturated-liquid points.

For alkanes larger than ethane, the presence of a dipole moment (and therefore a nonzero $A_{\mu}$ ) complicates matters, because both the $A_{\mu}$ and $a_{1}$ terms contribute temperature dependence in the low-density limit. For $\mathrm{C}_{3} \mathrm{H}_{8}$, the well-known dipole moment [55] and accurate low-density data [5] are sufficient to separate these effects with some confidence. The lowdensity data are reproduced within $0.02 \%$. The liquid data show errors in the fit of up to $0.5 \%$; this may reflect the fact that the dipolar term in Eq. (5) is strictly applicable only for low densities, and its use for a liquid is an approximation. $\mathrm{C}_{3} \mathrm{H}_{8}$ was the only pure component for which the Kirkwood expression for the polarization $\left(P_{\mathrm{K}}\right.$, Eq. (2)) led to a superior fit.

For $n-\mathrm{C}_{4} \mathrm{H}_{10}$ and $i-\mathrm{C}_{4} \mathrm{H}_{10}$, the low-density data [47] are much more limited. For these fluids, $a_{1}$ was set by scaling its value for ethane, assuming that it was proportional to the magnitude of $A_{\varepsilon}$. Then the known value of the dipole moment allowed $a_{0}$ to be determined from the lowdensity data. Substantial data at liquid-like densities were available for both fluids, and for the most part were fitted to within $0.05 \%$.

For alkanes $\mathrm{C}_{5}$ and beyond, the only low-density data of which we are aware $[66,67]$ are rather old and not of great precision. Therefore, the parameter $a_{1}$ was scaled to the value for ethane in the same way as for the butanes. The parameter $a_{0}$ was estimated by extrapolating the values for 
the lower alkanes, assuming that for a sufficiently long chain each added $\mathrm{CH}_{2}$ group made a constant contribution to the polarizability. The value $D=2$ determined for $n$-butane was used for all the higher alkanes.

The available dielectric data for these higher alkanes are sparse, and different sources are often inconsistent. We chose to rely on the data of Scaife and Lyons [51] for $n$-alkanes because they covered the widest temperature range and also studied compressed liquids. They also used higher-purity materials than most other studies. The liquid alkane data of Champion et al. [68] are systematically lower in $P / \rho$ by $0.1 \%$ to $0.3 \%$ (corresponding to about $0.001-0.004$ in $\varepsilon$ ), except for $n$-hexane where the Champion data are higher by about $0.15 \%$. For $n$-hexane, the data of Stokes [69] agree closely with those of Scaife and Lyons, while the data of Mopsik [70] are systematically higher by about $0.1 \%$. So, while we were generally able to fit the data of Scaife and Lyons [51] to within $0.05 \%$ in $P / \rho$, the inconsistency among data sources means that the actual uncertainty for these systems is somewhat greater. For isopentane, the two available sources $[43,53]$ agree within about $0.05 \%$ which is also approximately the scatter in the fit to the data.

In addition, the lack of low-density data on which to base $A_{\varepsilon}$ for the higher alkanes (and also the estimation required for their dipole moments) means that the values of $B_{\varepsilon}$ and $C$ reported for longer alkanes in Table II should not be considered to have physical significance.

\section{MIXTURE CALCULATIONS}

\subsection{Theory}

The classical way to represent the polarization of a mixture when pure-component values are available is due to Oster [71];

$$
(P / \rho)_{\operatorname{mix}}=\sum_{i} x_{i}\left(\frac{P(T, p)}{\rho(T, p)}\right)_{i}
$$

where $x_{i}$ is the mole fraction of component $i$. This becomes a linear volume-fraction mixing rule if the excess volume of mixing $V^{\mathrm{E}}$ at $(T, p)$ is zero;

$$
P_{\text {mix }}=\sum_{i} \Phi_{i} P_{i}(T, p) \quad\left[V^{\mathrm{E}}=0\right],
$$

where $\Phi_{i}=x_{i} v_{i} / \sum_{j} x_{j} v_{j}$ is the volume fraction of component $i$, with $v_{j}$ the molar volume of pure fluid $j$ at $(T, p)$. Equation (8), with Kirkwood's expression (Eq. (2)) used to relate $P$ to $\varepsilon$, was used by Wang and Anderko [72] to calculate the dielectric constants of mixtures of liquid solvents. 
A deficiency of Oster's rule (Eq. (7)) is its mixing of pure-component polarizations at constant temperature and pressure. While this is fine for liquid mixtures, for mixtures of components of greatly differing volatilities the environment of the pure component at $(T, p)$ may be very different from its environment in the mixture at the same $(T, p)$. For example, a heavy component in a gas mixture might be a liquid at $(T, p)$, and its molar polarization in that state might not be appropriate to describe its contribution to the polarization of a gas mixture.

Instead of mixing at constant temperature and pressure, Harvey and Prausnitz [6] proposed mixing at constant temperature and reduced density. Their mixing rule is

$$
P_{\mathrm{mix}}=\sum_{i} \Phi_{i}^{*} P_{i}\left(T, \frac{\rho_{\mathrm{r}, \mathrm{mix}}}{v_{i}^{*}}\right),
$$

where $v_{i}^{*}$ is a characteristic molar volume (here taken as the critical volume) for component $i, \Phi_{i}^{*}=x_{i} v_{i}^{*} / \sum_{j} x_{j} v_{j}^{*}$ is a volume fraction defined with these characteristic volumes, and $\rho_{\mathrm{r} \text {,mix }}$ is the dimensionless reduced molar density of the mixture,

$$
\rho_{\mathrm{r}, \text { mix }}=\rho_{\text {mix }} \sum_{i} x_{i} v_{i}^{*} .
$$

Because we anticipate extending this framework to polar components such as refrigerants, we use the Kirkwood expression, Eq. (2), to relate $P$ and $\varepsilon$ on both sides of Eq. (9). If one is using Eq. (2) in the "reverse" direction (from $P$ to $\varepsilon$ ), the solution is

$$
\varepsilon=\frac{1}{4}\left(1+9 P_{\mathrm{K}}+3 \sqrt{9 P_{\mathrm{K}}^{2}+2 P_{\mathrm{K}}+1}\right) .
$$

The procedure for computing the mixture dielectric constant at a given temperature, mixture density, and composition then consists of these steps:

(a) Compute $\rho_{\mathrm{r}, \mathrm{mix}}$ with Eq. (10).

(b) Compute the dielectric constant $\varepsilon$ for each component $i$ at temperature $T$ and density $\rho_{\mathrm{r} \text {,mix }} / v_{i}^{*}$. This uses the pure-component correlations (Eq. (5)) and also Eq. (1) or (11) to convert $P$ to $\varepsilon$, depending on whether $P_{\mathrm{CM}}$ or $P_{\mathrm{K}}$ was used in the correlation for component $i$.

(c) For each component, use Eq. (2) to calculate $P_{i}$ from the value of $\varepsilon$ determined in step (b). 


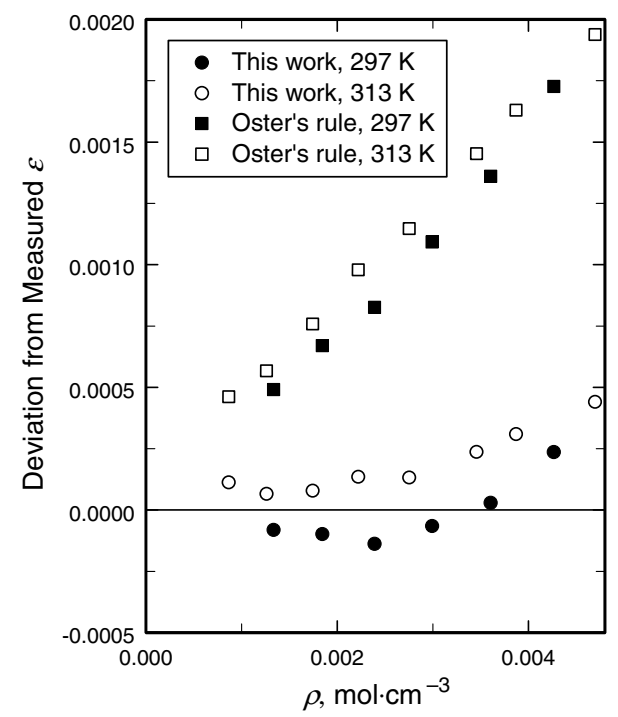

Fig. 3. Differences between predicted and measured values of the dielectric constant $\varepsilon$ for the methane/propane mixture studied by May et al. [73]. The reported experimental uncertainty in $\varepsilon$ is 0.0001 .

(d) Compute $P_{\text {mix }}$ with Eq. (9).

(e) Use Eq. (11) to compute the mixture dielectric constant $\varepsilon_{\text {mix }}$ from $P_{\text {mix }}$.

Often the pressure, not the density, will be known, in which case a mixture EOS must be used to calculate the density prior to the five steps above; the quality of the dielectric-constant prediction will depend strongly on the quality of the density prediction.

\subsection{Mixture Results}

We illustrate the performance of Eq. (9) by comparing its results (and results from Oster's rule, Eq. (7)) to data for mixtures of small hydrocarbons.

Figure 3 compares predictions for the methane/propane $(0.8419 / 0.1581$ mole fractions) mixture measured in the vapor phase by May et al. [73]. The Harvey-Prausnitz mixing rule (Eq. (9)) reproduces the measured values of the dielectric constant $\varepsilon$ very well; the errors for both isotherms are 


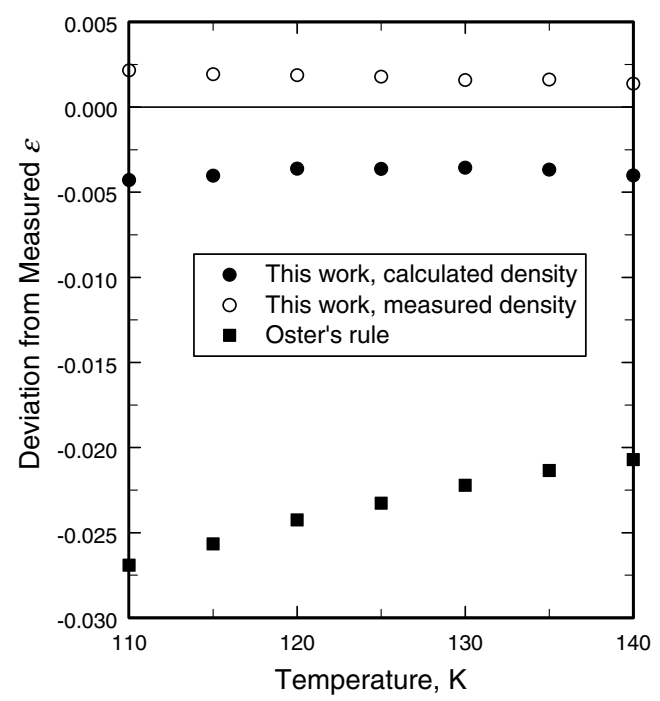

Fig. 4. Differences between predicted and measured values of the dielectric constant $\varepsilon$ for the methane/isobutane mixture studied by Haynes [74]. The reported experimental uncertainty is $0.05 \%$ in $\varepsilon$, which for these data corresponds to an uncertainty near 0.001 .

not much larger than the reported experimental uncertainty of 0.0001 in $\varepsilon$. Oster's rule (Eq. (7)) shows a larger systematic deviation, probably due to the fact that pure propane is a liquid at the temperature and pressure of the experiments, while the mixture is a vapor. Similar results are obtained for the two other dense vapor mixtures (ternary mixtures of methane, propane, and $n$-hexane) studied by May et al. [73].

Figure 4 shows a similar comparison for a methane/isobutane $(0.78329 / 0.21761$ mole fractions) mixture measured by Haynes [74] in the liquid phase at its bubble point. Again, Eq. (9) performs significantly better. If the mixture density is calculated with REFPROP [54], the improvement is approximately a factor of 5 over Oster's rule. If the actual densities measured by Haynes [74] are used, the error can be reduced still further (becoming comparable to the reported experimental uncertainty in $\varepsilon$ of approximately 0.001 ); this illustrates the importance of accurate density prediction as part of a model for the dielectric constant. Similar results to those shown in Fig. 4 are obtained for the five other binary mixtures (methane/isobutane and methane/ $n$-butane) studied by Haynes [74] and 
for the 17 multicomponent liquid mixtures (light hydrocarbons and, in some cases, nitrogen) studied by Haynes and McCarty [75].

\subsection{Possible Enhancement with Binary Parameters}

While Eq. (9) seems sufficiently accurate for the natural-gas systems considered here, such "ideal" mixing may be inadequate for other mixtures, particularly those involving polar components such as refrigerants or water.

Harvey and Prausnitz [6] proposed a modification of Eq. (9) in which the linear sum was replaced by a quadratic sum with an adjustable binary parameter for each pair. While their modified equation was able to correlate mixtures of polar liquids, it does not reduce to the correct low-density limit, where molecular interactions vanish and the polarization must be simply the sum of pure-component values.

There are at least two approaches that might be taken to fix this problem. First, the binary parameters might be applied only to those parts of the polarization beyond the $A_{\varepsilon}$ term, so that their influence would vanish at low densities. Second, the binary parameters themselves could be made density-dependent, with the boundary condition that they go to zero at zero density.

\section{CONCLUSIONS}

We have developed correlations for the static dielectric constant of a number of species, with special attention to those encountered in natural gas. These correlations improve on previous work due to the availability of better data at low densities, the availability of modern reference-quality equations of state for the pure fluids, and a functional form where the temperature dependence of the first and second dielectric virial coefficients is suggested by molecular theory.

To predict the dielectric constant of a mixture, we propose mixing the pure-component polarizations at constant temperature and reduced density. Comparison to literature data for mixtures of light hydrocarbons indicates that this is superior to the traditional method of mixing at constant temperature and pressure.

The pure-component correlations and mixing rule reported here will be implemented in an upcoming version of our database for mixture thermophysical properties [54] that is being developed with an emphasis on the properties of natural gas. 


\section{ACKNOWLEDGMENTS}

We thank E. F. May, M. R. Moldover, and J. W. Schmidt for extensive discussions and for sharing data from Refs. [5] and [30] prior to publication, W. Wagner for making Refs. [41] and [49] available prior to publication, A. Salam and K. K. Irikura for supplying ab initio calculations for $n$-pentane and isopentane, respectively, and M. Salehi for assistance with data entry.

\section{REFERENCES}

1. M. Jaeschke, Thermochim. Acta 382:37 (2002).

2. M. Jaeschke, P. Schley, and R. Janssen-van Rosmalen, Int. J. Thermophys. 23:1013 (2002).

3. B. A. Younglove and J. F. Ely, J. Phys. Chem. Ref. Data 16:577 (1987).

4. M. R. Moldover and T. J. Buckley, Int. J. Thermophys. 22:859 (2001).

5. J. W. Schmidt and M. R. Moldover, Int. J. Thermophys. 24:375 (2003).

6. A. H. Harvey and J. M. Prausnitz, J. Solution Chem. 16:857 (1987).

7. C. J. F. Böttcher, Theory of Electric Polarization, 2nd Ed., Vol. 1 (Elsevier, Amsterdam, 1973).

8. R. P. Bell, Trans. Faraday Soc. 38:422 (1942).

9. A. D. Buckingham and J. A. Pople, Trans. Faraday Soc. 51:1029 (1955).

10. M. Lallemand and D. Vidal, J. Chem. Phys. 66:4776 (1977).

11. R. D. McCarty and V. D. Arp, Adv. Cryog. Eng. 35:1465 (1990).

12. J. Huot and T. K. Bose, J. Chem. Phys. 95:2683 (1991).

13. V. V. Pashkov, M. P. Lobko, and V. S. Marinin, Sov. J. Low Temp. Phys. 13:70 (1987).

14. R. S. Katti, R. T Jacobsen, R. B. Stewart, and M. Jahangiri, Adv. Cryog. Eng. 31:1189 (1986).

15. W. P. Pan, M. H. Mady, and R. C. Miller, AIChE J. 21:283 (1975).

16. S. P. Singh and R. C. Miller, J. Chem. Thermodyn. 10:747 (1978).

17. C. Tegeler, R. Span, and W. Wagner, J. Phys. Chem. Ref. Data 28:779 (1999).

18. R. L. Amey and R. H. Cole, J. Chem. Phys. 40:146 (1964).

19. D. Vidal, L. Guengant, P. Malbrunot, and J. Vermesse, Mol. Phys. 68:737 (1989).

20. E. W. Lemmon and R. Span, submitted to J. Chem. Eng. Data.

21. J. van der Elsken and J. C. F. Michielsen, Chem. Phys. Lett. 115:230 (1985).

22. J. W. Stewart, J. Chem. Phys. 40:3297 (1964).

23. B. A. Younglove, J. Phys. Chem. Ref. Data 11 (Suppl. 1):1 (1982).

24. J. F. Ely and G. C. Straty, J. Chem. Phys. 61:1480 (1974).

25. R. Span, E. W. Lemmon, R. T Jacobsen, W. Wagner, and A. Yokozeki, J. Phys Chem. Ref. Data 29:1361 (2000).

26. B. A. Younglove, J. Res. Nat. Bur. Stand. A 76A:37 (1972).

27. R. Schmidt and W. Wagner, Fluid Phase Equilib. 19:175 (1985).

28. T. Moriyoshi, T. Kita, and Y. Uosaki, Ber. Bunsenges. Phys. Chem. 97:589 (1993).

29. J. Obriot, J. Ge, T. K. Bose, and J.-M. St-Arnaud, Fluid Phase Equilib. 86, 315 (1993).

30. E. F. May, M. R. Moldover, and J. W. Schmidt, Int. J. Thermophys., in press.

31. R. Span and W. Wagner, J. Phys. Chem. Ref. Data 25:1509 (1996).

32. G. C. Straty and R. D. Goodwin, Cryogenics 13:712 (1973).

33. T. Vienravee and R. C. Miller, J. Chem. Thermodyn. 14:361 (1982). 
34. J.-M. St-Arnaud, A. Hourri, T. K. Bose, and D. Ingrain, High Temp. High Press. 25:301 (1993).

35. P. Malbrunot, J. Vermesse, D. Vidal, T. K. Bose, A. Hourri, and J. M. St-Arnaud, Fluid Phase Equilib. 96:173 (1994).

36. U. Setzmann and W. Wagner, J. Phys. Chem. Ref. Data 20:1061 (1991).

37. T. K. Bose and R. H. Cole, J. Chem. Phys. 54:3829 (1971).

38. W. M. Haynes, Cryogenics 25:68 (1985).

39. J. Smukala, R. Span, and W. Wagner, J. Phys. Chem. Ref. Data 29:1053 (2000).

40. L. A. Weber, J. Chem. Phys. 65:446 (1976).

41. D. Bücker and W. Wagner, J. Phys. Chem. Ref. Data (in press).

42. R. T. Thompson, Jr. and R. C. Miller, Adv. Cryog. Eng. 25:698 (1980).

43. C. C. Luo and R. C. Miller, Cryogenics 21:85 (1981).

44. W. M. Haynes and B. A. Younglove, Adv. Cryog. Eng. 27:883 (1982).

45. W. M. Haynes, J. Chem. Thermodyn. 15:419 (1983).

46. E. W. Lemmon, M. O. McLinden, and W. Wagner, to be submitted to J. Phys. Chem. Ref. Data.

47. H. E. Watson and K. L. Ramaswamy, Proc. Roy. Soc. A 156:144 (1936).

48. W. M. Haynes, J. Chem. Thermodyn. 15:801 (1983).

49. D. Bücker and W. Wagner, J. Phys. Chem. Ref. Data (in press).

50. W. M. Haynes, J. Chem. Eng. Data 28:367 (1983).

51. W. G. S. Scaife and C. G. R. Lyons, Proc. R. Soc. Lond. A 370:193 (1980).

52. R. Span and W. Wagner, Int. J. Thermophys. 24:41 (2003).

53. F. I. Mopsik, J. Chem. Phys. 50:2559 (1969).

54. E. W. Lemmon, M. O. McLinden, and M. L. Huber, Reference Fluid Thermodynamic and Transport Properties, NIST Standard Reference Database 23, Version 7.0 (Nat. Inst. Stands. Technol., Gaithersburg, Maryland, 2002).

55. R. D. Nelson, Jr., D. R. Lide, Jr., and A. A. Maryott, Selected Values of Electric Dipole Moments for Molecules in The Gas Phase, National Standard Reference Data Series, NSRDS-NBS 10 (U.S. Government Printing Office, Washington, D.C., 1967).

56. W. Hüttner, W. Majer, and H. Kästle, Mol. Phys. 67:131 (1989).

57. W. A. Herrebout, B. J. van der Veken, A. Wang, and J. R. Durig, J. Phys. Chem. 99:578 (1995).

58. A. Salam and M. S. Deleuze, J. Chem. Phys. 116:1296 (2002).

59. A. Salam, Department of Chemistry, Wake Forest University, Winston-Salem, North Carolina, personal communication (2004).

60. K. K. Irikura and A. H. Harvey, unpublished.

61. W. Cencek, K. Szalewicz, and B. Jeziorski, Phys. Rev. Lett. 86:5675 (2001).

62. J. Rychlewski, Mol. Phys. 41:833 (1980).

63. M. B. Ewing and D. D. Royal, J. Chem. Thermodyn. 34:1089 (2002).

64. M. B. Ewing and D. D. Royal, J. Chem. Thermodyn. 34:1985 (2002).

65. W. M. Haynes, Adv. Cryog. Eng. 31:1199 (1986).

66. C. P. Smyth and K. B. McAlpine, J. Am. Chem. Soc. 56:571 (1934).

67. M. Kubo, Sci. Pap. Inst. Phys. Chem. Res. Jpn. 26:242 (1935).

68. J. V. Champion, G. H. Meeten, and C. D. Whittle, Trans. Faraday Soc. 66:2671 (1970).

69. R. H. Stokes, J. Chem. Thermodyn. 5:379 (1973).

70. F. I. Mopsik, J. Res. Nat. Bur. Stand. A 71A:287 (1967).

71. G. Oster, J. Am. Chem. Soc. 68:2036 (1946).

72. P. Wang and A. Anderko, Fluid Phase Equilib. 186:103 (2001).

73. E. F. May, R. C. Miller, and A. R. H. Goodwin, J. Chem. Eng. Data 47:102 (2002).

74. W. M. Haynes, J. Chem. Thermodyn. 15:903 (1983).

75. W. M. Haynes and R. D. McCarty, Cryogenics 23:421 (1983). 